\title{
Upper Extremity Kinetics during Walker-Assisted Gait of Knee Joint Stiffness Simulation
}

\author{
Dong Ming*, Xiuyun Liu, Yanru Bai, Xingwei An, \\ Hongzhi Qi, Baikun Wan \\ Department of Biomedical Engineering \\ Tianjin University \\ Tianjin, China \\ *: richardming@tju.edu.cn
}

\author{
Yong $\mathrm{Hu}$, KDK Luk \\ Department of Orthopaedics and Traumatology \\ University of Hong Kong \\ Hong Kong, China
}

\begin{abstract}
Because of various inflammations and fractures of low limbs, the number of patients with knee joint stiffness is increasing. Walkers are commonly prescribed to improve these patients' stability and ambulatory ability. The evaluation on the assisted walking effect has become a hot problem, whose prerequisite is a comprehensive mechanical understanding of the upper extremity force. In order to study the upper extremity kinetics during walker-assisted gait of knee joint stiffness, this paper developed a new method to collect upper extremity kinetics data based on a special-designed walker dynamometer system. Handle reaction vector (HRV) data were collected from 15 healthy right-handed young subjects by simulation experiments for four knee joint stiffness modes. $T$ test and support vector machine (SVM) were used to analyze these HRV data. The results indicated that knee joint stiffness had a great influence on the upper extremity force during the walker-assisted walking. The proposed method is hoped to beneficially influence walkerassisted gait retraining strategies for knee joint stiffness.
\end{abstract}

Keywords- knee joint stiffness;walker;gait; handle reaction vector; support vector machine

\section{INTRODUCTION}

As known, the knee is one of the most important joints of human body. It plays a great deal of roles, such as transferring body load, keeping momentum, offering kinetic strength to the legs and so on. Nowadays, because of various inflammations of low limbs, as well as fractures, the number of patients with knee joint stiffness increases year by year. It has been reported that, even in the western countries with developed medical treatments, the incidence of stiffness reached $11 \%$ among the knee joint diseases [1]. Thus knee joint stiffness has become a social problem that needs to be solved urgently. To help these patients regain their normal walking ability is a significant job in rehabilitative field.

As a rehabilitation aid tool, the walker provides external mechanical support and reduces weight bearing on the lower limbs. Walker has been used more often than any other mobility aids, second only to the cane [2]. It is most helpful to patients who suffer from knee joint stiffness. In the kinetic analysis of walker-assisted gait, the upper extremities forces of handle reaction vector (HRV) are necessary to quantify assistive demands of patients within the gait cycle [2-3]. The potential clinical benefit of HRV measurement and analysis is that it may provide some criterions for the evaluation and classification of the walking efficiency with walker usage for some diseases, like knee joint stiffness.

The present measurement and analysis system for HRV described in the literature was designed to provide direct three degree-of-freedom load measurement [4], which was discomfortable for the users and their normal gaits may be influenced or even distorted. Therefore quantification of left and right upper extremity loads could not be exactly collected and full kinetic analysis of upper extremities in walkerassisted gait was not reliable.

This paper developed a new method to collect upper extremities forces of HRV in knee joint stiffness simulation experiment based on a special-designed walker dynamometer system. By using methods of $T$ tests and support vector machine (SVM), a significant difference analysis of HRV characteristic parameters was carried out. Another specific objective of our research was to find out whether different knee stiffness modes have different effects on upper limb forces on walker. It was hoped that our description of HRV in upper extremity kinetics may beneficially influence gait retraining strategies for knee joint stiffness.

\section{MATERIALS AND METHODS}

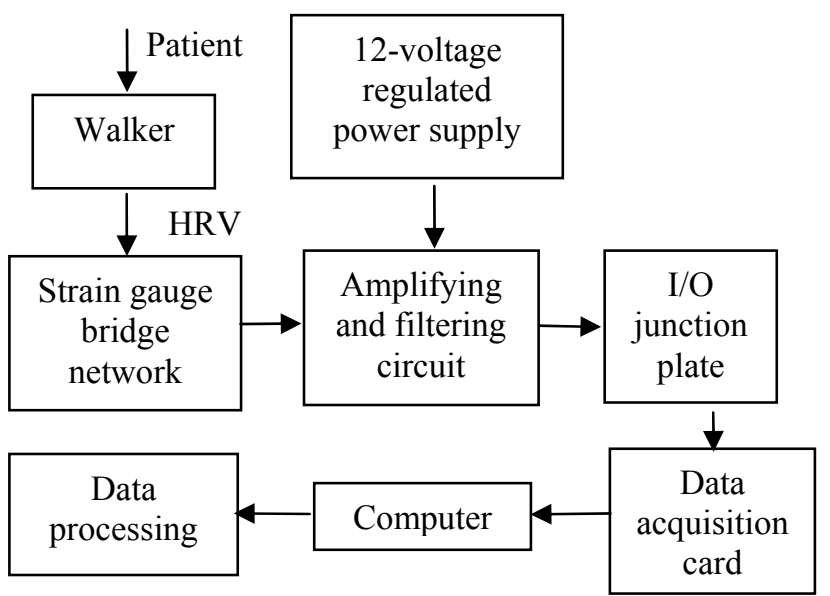

Figure 1. Schematic representation of walker dynamical system 
In this paper, the system consisted of a strain gauge-based walker and data collection and analysis system. In other words, it contained two parts: hardware and software. The former part consisted of the sensors, the amplifying and filtering circuit, the regulated power supply and the digital-analog conversion device. The latter one was constituted by data acquisition system and processing system. A schematic representation of the whole system was given in Fig. 1.

\section{A. Walker dynamometer system}

12 strain gauge bridges were mounted on walking frame to detect HRV forces in 3-dimension, that were rightward ( $x$-axis), forward ( $y$-axis) and upward ( $z$-axis) directions. Twelve strain gauge bridges (BX350-6AA) were employed to be the sensors of this walker. The positions of these strain gauge bridges were determined according to the bending moment distributions on the walker frame, which were calculated using the finite element analysis (FEA) by MARC finite element software (MARC Analysis Research Corporation, Palo Alto, CA). The strain gauge bridges were connected with the following circuit through four-core data transmission cables, among which two were used for power supply and other two for signal transmission. To implement the dynamical assessing system, the amplifying and filtering circuit was necessary to translate the weak signal into the voltage signal that was adapted for the following data acquisition.

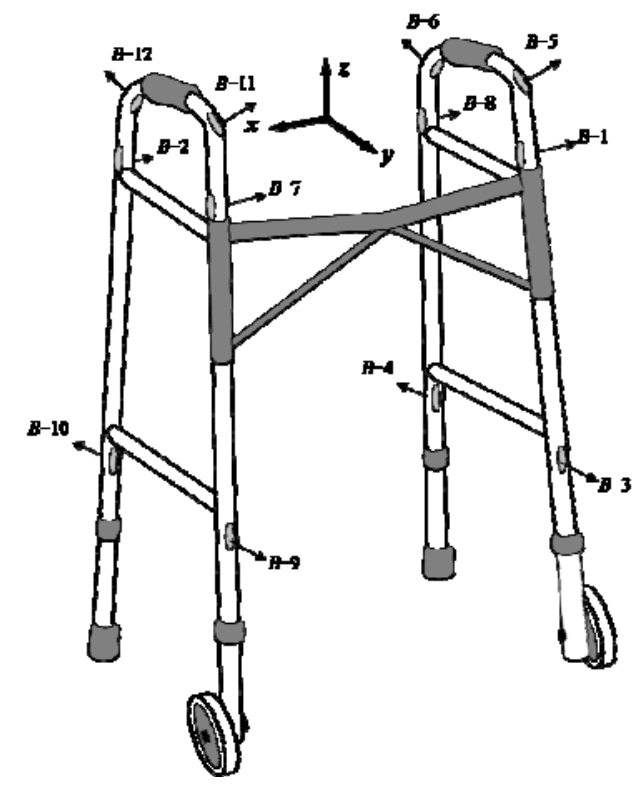

Figure 2. 12 strain gauge-bridge positions on walker frame

In this research, we chose regulated power supply whose output voltage ranged from -12 to $12 \mathrm{~V}$. The digital- analog conversion device consisted of I/O junction plate and multifunction data acquisition card. The junction plate (CB-68LPR) could offer the low-noise signal as input of data acquisition card. The NI6024E was used as multi-function data acquisition card. It had a 16-channel single-ended/8-channel analog input, 12 bit sampling precision, $200 \mathrm{KS} / \mathrm{s}$ sampling rate and the same disk write speed. In addition, it had 2-channel 24 bit counter/timer, at most 2-channel 12 bit analog output and 32 digital I/O wires. Hence, it could meet the request of the experiment. In addition, we chose PSHR68-68 shielding wires as the link between I/O junction plate and data acquisition card. Then, the signal from digital-analog convertor could enter the computer to be saved and processed.

Data acquisition software was established based on LabVIEW (Version 7.0, NI) which was a virtual software exploitation platform and had a powerful function module database. Using this software in the research, we could gain and analyze the real-time data. The program was made up of two parts. The front panel formed an interactive humanmachine interface, through which we could build the control panel of the real instrument, as well as modulate the sampling rate and sampling length. After being collected, data was displayed in the waveform graph and saved in terms of txt file format so that we could test errors on the channels and analyze the data in the following work. The latter panel was block diagram corresponding to the front panel.

To calculate hand loads represented by HRV from the output voltages of the 12 strain gauge bridges, the walker was statically calibrated using a multi-axis frame designed to apply known loads, known forces in another word, in different directions. And to evaluate the force measurement reliability, system performance was testified via nonlinearity validation, crosstalk test, and force measurement accuracy check, which were found to be better than $1.01 \%, 2.90 \%$, and $3.19 \%$, respectively [5]. These data meant that the implemented walker system was reliable for the measurement of hand loads and the description of kinetic analysis of walker-assisted gait.

\section{B. Walking experiment}

In the knee joint stiffness simulation experiments, 15 healthy right-handed youths were chosen as subjects, containing 10 males and 5 females whose age ranged from 21.2 to 25 years old. Their heights were from 161.1 to 178.1 $\mathrm{cm}$, with the weights from 51.14 to $81.26 \mathrm{~kg}$. All the subjects had never used the walker before and agreed to participate in the experiment. The subject's knee was immobilized with a long-leg knee-immobilizer to keep similar gait state of completely extended knee to knee joint stiffness. In the process of experiment, each subject was requested to walk in four modes that contained left knee fixed $(L)$ mode, right knee $(R)$ fixed mode, both knees fixed mode $(B)$ and no knees fixed (N) mode.

The subjects were asked to walk in the way of four-point gait as shown in Fig.3. The gait cycle included the events as follows: move the walker; stop the walker; move one foot; stop the foot; move the other foot; stop the foot; move the walker [6-8].

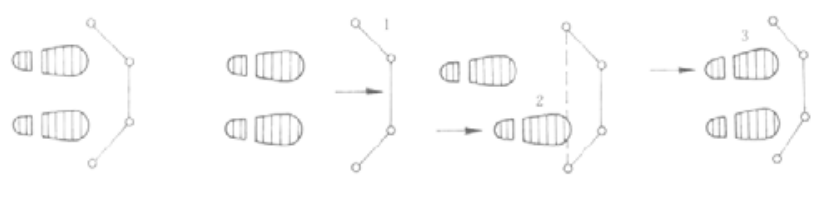

Figure 3. Sketch map of four-point gait 
When the test starts, the subject would walk in a walkway with the length of 10 miles, using the walker dynamical system. To offer the natural data of upper limb force, the subject himself control his walking rhythm. In each test, subject kept walking at least 5 standard gait cycles. They repeated each mode test 3 times. Before data collection, the walker dynamometer system was powered for 10 minutes to allow instrumentation temperature stabilization. The essential initialization measurement of the system was introduced in order to eliminate the influence of the circumstance.

\section{Statistics analysis}

With the development of testing instrument and the increasing of testing sample, statistics was applied to help us find the discipline of human body movement from the exterior multifarious data. On the basis of the statistical analysis, a SVM pattern recognition investigation of different stiffness modes was made in this study by using HRV as the eigenvector.

As mentioned above, we could get the data of 15 standard gait cycle of each subject. By way of eliminating the difference among subjects, normalization processing was applied on the gait parameters before statistics analysis. In this paper, vertical component force is divided by half the weight of relevant subject to avoiding the difference caused by their weights; To eliminate the error due to the different walking speed, the interval of one gait cycle was converted to the percentage of unit gait cycle.

\section{1) $\quad T$ test}

Considering the difference of gait, we chose coefficient of variation (CV) to evaluate variability of the data of upper limb force, which was shown as [9-10]:

$$
C V=\frac{S . D .}{\text { Mean }} \times 100 \%
$$

When $|C V|<12.5 \%$, the variability could be acceptable; Otherwise, it couldn't be accepted.

$T$ test was a method used for the different degree of two mean values in a small swatch whose number was less than 30 The steps were as follows: Firstly, build up hypothesis, supposing that there were no difference exists between the two samples. Secondly, calculate $T$ value. Here, it was calculated as equation (2). Thirdly, according to the degree of freedom, consult the theoretical value of $T$ and compare them. Finally, analyze the probability of occurrence and get the conclusion.

$$
T=\frac{\overline{X_{1}}-\overline{X_{2}}}{\sqrt{\frac{\sum x_{1}^{2}+\sum x_{2}^{2}}{n_{1}+n_{2}-2} \times \frac{n_{1}+n_{2}}{n_{1} \times n_{2}}}}
$$

\section{2) Support Vector Machine( SVM)}

The Support Vector Machine (SVM) developed by V. Vapnik of AT\&T Bell lab was a method aimed at solving classific and recursive problems. It has complete statistical learning theory and could avoid the disadvantages of neural networks, such as complexity, limitation, etc. So recently, SVM has been widely used in academe.

The goal of SVM is to produce a model which predicts target value of data instances in the testing set that are given only the attributes. After mapping the vector into a higher (maybe infinite) dimensional space by the kernel function, SVM finds a linear separating hyperplane with the maximal margin in this higher dimensional space. It has four basic kernels that are linear, polynomial, radial basis function (RBF) and sigmoid.

In this research, we selected RBF as our kernel function. The basic procedure was as follows: Transform data to the format of an SVM software; Conduct simple scaling on the data; Consider the RBF kernel ; Use cross-validation to find the best parameter $\mathrm{C}$ and $\mathrm{g}$; Use the best parameters to train the whole training set; Test and get conclusion [11-12].

\section{RESULTS}

The curves of upper limb force at unit gait cycle were given in Fig.4 to Fig. 9. The forces were depicted in turn of $F_{1 x}$, $F_{l y}, F_{l z}, F_{r x}, F_{r y}, F_{r z}$. And in each figure, the forces of four patterns were given to do comparison and statistics analysis. Proceeding from these six figures, we can find that that in the horizontal and vertical directions, average mean was positive at the preceding one-third gait cycle and negative during the later two-thirds. At the same time, average mean was basically positive at the whole gait cycle in the posterior direction. And they had the maximum as in the figure, respectively. Comparing the relationship between each force component and gait cycle, we could see there was a significant amplitude changing in patients' upper extremity strength at the region of $30 \%$ to $60 \%$ of gait cycle, which interval was just the suspend period of uplifting and falling the right foot. In addition, we found the curves of $F_{\mathrm{l}}, F_{\mathrm{lz}}$ and $\mathrm{F}_{\mathrm{rz}}$ were similar between left mode and both mode. In the same way, the similarity existed between right mode and both mode. In the other three directions, the curves were obviously different.

We adopted $T$ test to find the degree of difference of characteristic parameters through comparing and analyzing two different patterns. The feature parameters were described as $F_{\mathrm{i}}$, which denoted the component force of HRV. If $T$ value was less than 0.05 , that meant significant difference existed between the two modes. Table 1 shown as follows, gave the result of comparison among these four modes. We could see that between the left knee fixed mode and right knee fixed mode, there was obvious difference for all six components of HRV, and the same was true of both knees stiffness and normal pattern.

By the means of SVM, we made the pattern recognition of different stiffness modes by using HRV as the eigenvector. From Table 2, we could see the accuracy of distinguishing different patterns were all higher than $85 \%$, with its highest value of $100 \%$. These results indicated that SVM was suitable for separating different stiffness modes and the study also make it clear that knee stiffness have a great effect on the upper extremity force. 


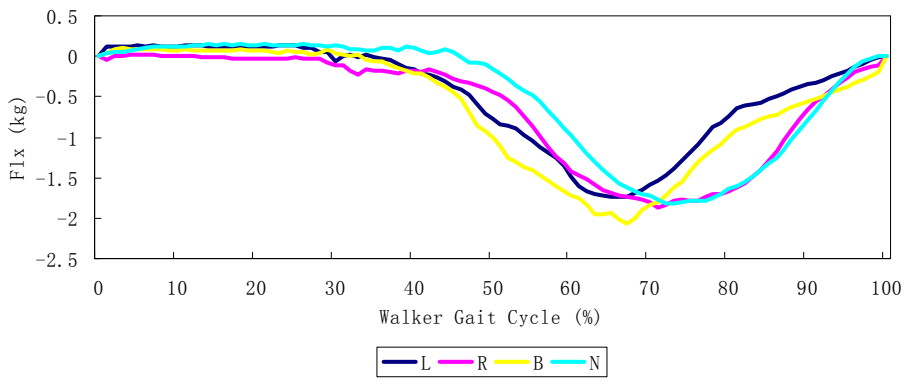

Figure 4. The curves of HRV-Flx in one walker gait cycle

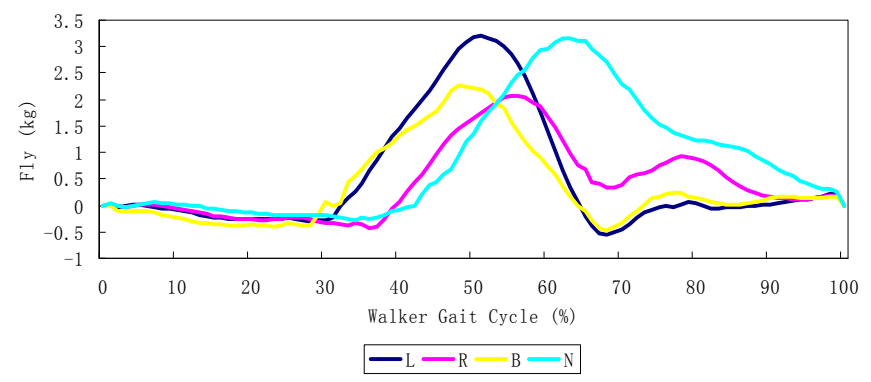

Figure 5. The curves of HRV-Fly in one walker gait cycle

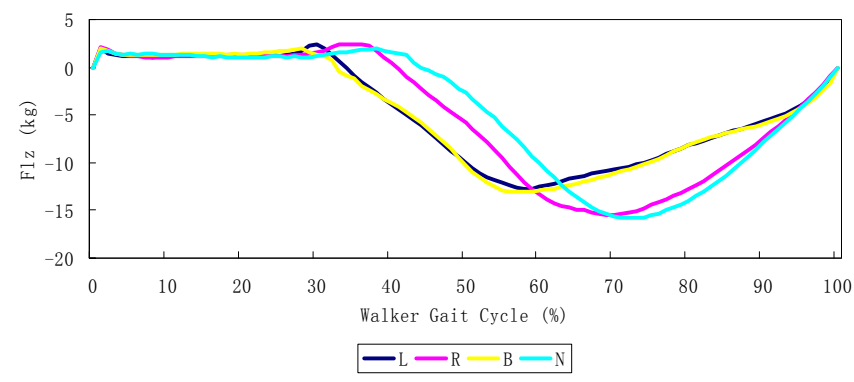

Figure 6. The curves of HRV-Flz in one walker gait cycle

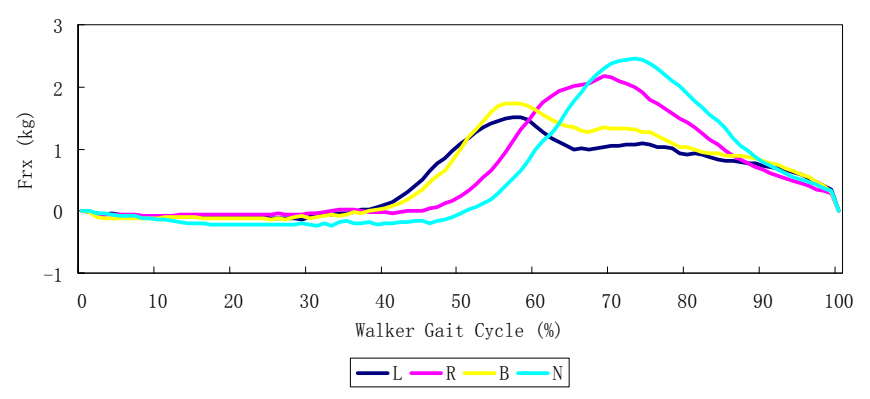

Figure 7. The curves of HRV-Frx in one walker gait cycle

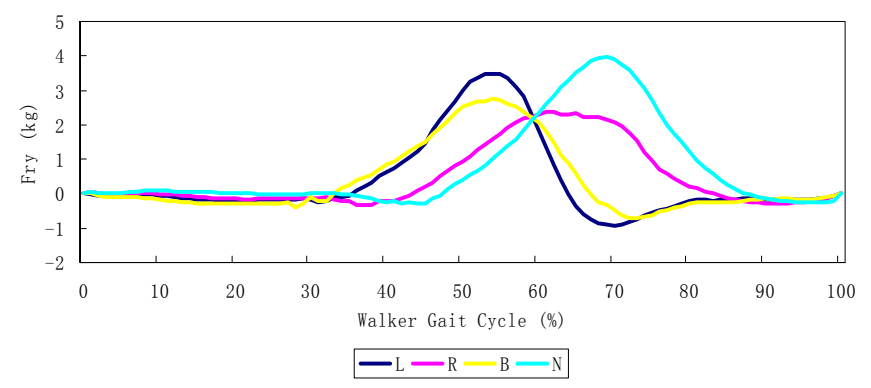

Figure 8. The curves of HRV-F $\mathrm{F}_{\mathrm{ry}}$ in one walker gait cycle

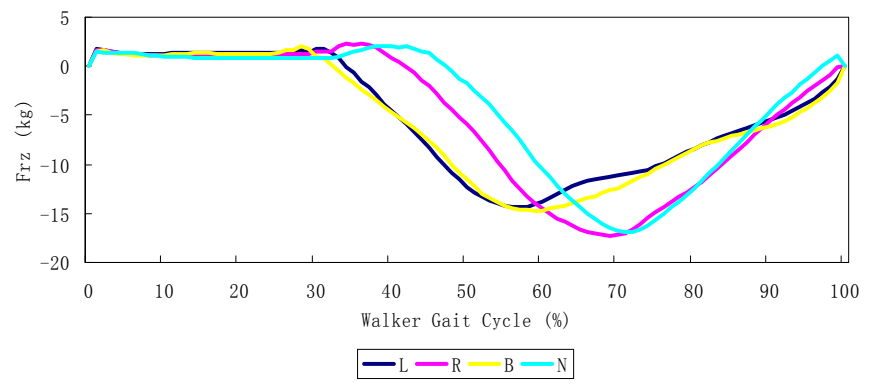

Figure 9. The curves of HRV-F $\mathrm{F}_{\mathrm{rz}}$ in one walker gait cycle

TABLE I. T-VALUE OF STATISTICS ANALYSIS FOR FOUR MODES

\begin{tabular}{|c|c|c|c|c|}
\hline \multirow{2}{*}{ HRV } & \multicolumn{4}{|c|}{ T-value } \\
\cline { 2 - 5 } & $\boldsymbol{L \&} \boldsymbol{N}$ & $\boldsymbol{R \&} \boldsymbol{N}$ & $\boldsymbol{L \&} \boldsymbol{R}$ & $\boldsymbol{B} \boldsymbol{\&} \boldsymbol{N}$ \\
\hline Flx & 0.0091 & 0.3284 & 0.0021 & 0.0068 \\
\hline Fly & 0.2954 & $7.17 \mathrm{E}-10$ & $7.01 \mathrm{E}-11$ & $3.71 \mathrm{E}-08$ \\
\hline Flz & $2.28 \mathrm{E}-07$ & 0.0501 & $2.74 \mathrm{E}-06$ & $5.02 \mathrm{E}-05$ \\
\hline Frx & $2.76 \mathrm{E}-08$ & 0.0265 & $6.53 \mathrm{E}-08$ & $2.87 \mathrm{E}-08$ \\
\hline Fry & 0.0092 & $2.02 \mathrm{E}-10$ & $1.71 \mathrm{E}-10$ & $2.79 \mathrm{E}-08$ \\
\hline Frz & 0.0017 & 0.4793 & $7.61 \mathrm{E}-05$ & 0.0041 \\
\hline
\end{tabular}

TABLE II.

SVM ACCURACY OF DISTINGUISHING MODEES

\begin{tabular}{|c|c|c|c|c|}
\hline \multirow{2}{*}{ HRV } & \multicolumn{4}{|c|}{ Accuracy } \\
\cline { 2 - 5 } & $\boldsymbol{L} \boldsymbol{\&} \boldsymbol{N}$ & $\boldsymbol{R} \boldsymbol{N}$ & $\boldsymbol{L} \boldsymbol{R}$ & $\boldsymbol{B} \boldsymbol{\&} \boldsymbol{N}$ \\
\hline Flx & $93.33 \%$ & $90 \%$ & $96.67 \%$ & $100 \%$ \\
\hline Fly & $96.67 \%$ & $93.33 \%$ & $96.67 \%$ & $96.67 \%$ \\
\hline Flz & $96.67 \%$ & $86.67 \%$ & $96.67 \%$ & $100 \%$ \\
\hline Frx & $100 \%$ & $90 \%$ & $100 \%$ & $100 \%$ \\
\hline Fry & $100 \%$ & $93.33 \%$ & $96.67 \%$ & $100 \%$ \\
\hline Frz & $96.67 \%$ & $86.67 \%$ & $100 \%$ & $96.67 \%$ \\
\hline
\end{tabular}

\section{CONCLUSION}

In this study, we have performed knee joint stiffness simulation experiments on 15 healthy youths. After collecting the dynamical data of HRV, the methods of $T$ test and SVM were employed to do statistics analysis. The results indicated that knee joint stiffness may have a great influence on upper limb force and different knee stiff modes have obvious influence. The study also supported that SVM was a suitable tool for classifying stiffness modes. These results will be helpful to further evaluation of the effects of rehabilitation walking of knee joint stiffness.

\section{ACKNOWLEDGMENT}

This research was supported by National Natural Science Foundation of China (No.30970875, No.90920015 and No. 60501005), Joint Project of National Natural Science Foundation of China-Royal Society of Edinburgh of UK (No. 30910494/C1009), The National High Technology Research and Development Program of China (No.2007AA04Z236), Intentional Cooperation Key Program (No.08ZCGHHZ00300) and Biomedical Engineering Key Program (No. 07ZCKFSF01300) of Tianjin Science Technology Support Plan.

\section{REFERENCES}


[1] P.J. Millett, B. Johnson, J. Carlson, S. Krishnan, J.R. Steadman. "Rehabilitation of the arthrofibrotic knee", Am J Orthop. vol. 32, pp. 531-538, 2003.

[2] National Center for Health Statistics: "Number persons using assistive technology by age of person and type of device",1994.

[3] D. N, and Y. CH. "A study of handle reaction vector (HRV) in walker FES standing”, Proc Inst Mech Eng. vol.211, pp. 81-94, 1996.

[4] R.A. Bachschmidt, G.F. Harris and G.G. Simoneau. "Walker-assisted gait in rehabilitation: a study of biomechanics and instrumentation. IEEE Trans Neural Syst Rehabil Eng. vol. 9, pp. 96-105, 2001.

[5] D. Ming, B.K. Wan, Y. Hu. "A new dynamometer walker system for the measurement of handle reaction vector (HRV)". Meas Sci Technol. vol. 16, pp. 1272-1280, 2005.

[6] P.S. Requejo, D.P. Wahl, E.L. Bontrager, C.J. Newsam, J.K. Gronley, S.J. Mulroy, J. Perry. "Upper extremity kinetics during Lofstrand crutchassisted gait". Med Eng Phys. vol. 27(1), pp.19-29, 2005.
[7] J. Babic, T. Karcnik, T. Bajd. "Stability analysis of four-point walking". Gait Posture. Vol. 14, pp.56-60, 2001.

[8] T. Bajd, A. Kralj, M. Zefran. "Unstable states in four-point walking”. $J$ Biomed Eng. Vol.15, pp.159-162, 1993.

[9] K. Canseco, J. Long, R. Marks, M. Khazzam, G. Harris. "Quantitative characterization of gait kinematics in patients with hallux rigidus using the Milwaukee foot model". J Orthop Res. vol. 26, pp.419-427, 2008.

[10] E. Isakov. "Gait rehabilitation: a new biofeedback device for monitoring and enhancing weight-bearing over the affected lower limb". Eura Medicophys. vol. 43, pp. 21-26, 2007.

[11] Y. Mao, M. Saito, T. Kanno, D. Wei, H. Muroi. "Walking pattern analysis and SVM classification based on simulated gaits". Conf Proc IEEE Eng Med Biol Soc. pp.5069-5072, 2008;.

[12] S. S.Keerthi, and C. J. Lin. "A symptotic behaviors of support vector machines with Gaussian kernel". Neural Computation. vol. 15, pp. $1667-1689,2003$. 